\title{
Explicit Difference Methods for Solving the Cylindrical Heat Conduction Equation
}

\author{
By A. R. Mitchell and R. P. Pearce
}

1. Introduction. In the past, several authors have used finite difference methods to solve the cylindrical heat conduction equation

$$
\frac{\partial u}{\partial t}=\frac{1}{r} \frac{\partial u}{\partial r}+\frac{\partial^{2} u}{\partial r^{2}}, \quad(0 \leqq r \leqq 1)
$$

subject to appropriate boundary conditions. One of the most recent efforts was by Albasiny [1], who obtained a numerical solution of (1) for the problem of a cooling spinning thread line. The appropriate boundary conditions were

$$
u=1 \text { for all } r \text { at } t=0,
$$

and

$$
\frac{\partial u}{\partial r}=0(r=0), \quad \frac{1}{u} \frac{\partial u}{\partial r}=-F(t), \quad(r=1)
$$

where $F(t)$ is an empirical function of $t$.

In all cases, as far as the authors are aware, the differential coefficients in (1) are considered separately when replaced by differences, and the principal term in the truncation error of any difference formula obtained in this way contains $\frac{\partial u}{\partial r}$ and $\frac{\partial^{2} u}{\partial r^{2}}$. This can be seen by obtaining the time derivatives of $u$ from (1) in the form

$$
\begin{aligned}
& \frac{\partial u}{\partial t}=\frac{1}{r} \frac{\partial u}{\partial r}+\frac{\partial^{2} u}{\partial r^{2}}, \\
& \frac{\partial^{2} u}{\partial t^{2}}=\frac{1}{r^{3}} \frac{\partial u}{\partial r}-\frac{1}{r^{2}} \frac{\partial^{2} u}{\partial r^{2}}+\frac{2}{r} \frac{\partial^{3} u}{\partial r^{3}}+\frac{\partial^{4} u}{\partial r^{4}}, \\
& \frac{\partial^{3} u}{\partial t^{3}}=\frac{9}{r^{5}} \frac{\partial u}{\partial r}-\frac{9}{r^{4}} \frac{\partial^{2} u}{\partial r^{2}}+\frac{4}{r^{3}} \frac{\partial^{3} u}{\partial r^{3}}-\frac{3}{r^{2}} \frac{\partial^{4} u}{\partial r^{4}}+\frac{3}{r} \frac{\partial^{5} u}{\partial r^{5}}+\frac{\partial^{6} u}{\partial r^{6}}
\end{aligned}
$$

and noticing that $\frac{\partial u}{\partial r}$ and $\frac{\partial^{2} u}{\partial r^{2}}$ occur in all the time derivatives. Thus no matter how accurately $\frac{\partial u}{\partial t}$ is replaced by differences the error always contains the low order derivatives of $u$ with respect to $r$.

2. Transformation of the Equation. If the variable $r$ is transformed according to the relation

$$
r=2 x^{1 / 2}
$$

Received November 14, 1962. 
equation (1) becomes

$$
\frac{\partial u}{\partial t}=\frac{\partial u}{\partial x}+x \frac{\partial^{2} u}{\partial x^{2}} . \quad\left(0 \leqq x \leqq \frac{1}{4}\right) .
$$

The higher time derivatives of $u$ are given by

$$
\begin{aligned}
& \frac{\partial^{2} u}{\partial t^{2}}=2 \frac{\partial^{2} u}{\partial x^{2}}+4 x \frac{\partial^{3} u}{\partial x^{3}}+x^{2} \frac{\partial^{4} u}{\partial x^{4}} \\
& \frac{\partial^{3} u}{\partial t^{3}}=6 \frac{\partial^{3} u}{\partial x^{3}}+18 x \frac{\partial^{4} u}{\partial x^{4}}+9 x^{2} \frac{\partial^{5} u}{\partial x^{5}}+x^{3} \frac{\partial^{6} u}{\partial x^{6}},
\end{aligned}
$$

expressions which no longer contain the low order derivatives of $u$ with respect to $x$.

In what follow, the expressions (4) are used to obtain finite difference replacements of (3), and the accuracy of these formulas is tested by using them to solve the cylindrical heat conduction equation subject to the boundary conditions

$$
\begin{array}{rlrl}
u & =J_{0}(\alpha r) & (0 \leqq r \leqq 1) \text { at } t=0, \\
\frac{\partial u}{\partial r}=0(r=0), & u=0(r=1),
\end{array}
$$

where $\alpha$ is the first root of $J_{0}(\alpha)=0$. This is a perfectly straightforward problem and has the theoretical solution

$$
u=J_{0}(\alpha r) e^{-\alpha^{2} t}
$$

3. Explicit Formulas. It has been a feature of numerical solutions of (1) using finite differences on a rectangular net equally spaced in the $r$-direction that the solution has been less accurate in the vicinity of the axis than in the remainder of the field. This is presumably due to the term $\frac{1}{r} \frac{\partial u}{\partial r}$ in (1), which for adequate representation by finite differences, requires points closer together in the $r$-direction when $r$ is small. On the axis, of course, when $r=0$, the term becomes $\frac{\partial^{2} u}{\partial r^{2}}$.

In the $(x, t)$ plane, the rectangular net used to derive explicit replacements of (3) has equal spacing in the $t$-direction given by $t=j k(j=1,2,3, \cdots)$ and unequal spacing in the $x$-direction given by $x=i^{2} h(i=1,2,3, \cdots)$, where $h, k$ are constants. The mesh spacing is to some extent arbitrary and is chosen to suit the problem to be solved. The spacing suggested appears suitable for the problem stated where the only difficult region is in the neighborhood of the axis. It has the added advantage that the corresponding points for the $r$-coordinate are equally spaced.

The following procedure, suggested previously by the present authors $[2,3]$ in the solution by difference methods of other partial differential equations is used to derive optimum four and five point explicit difference replacements of (3). Consider initially the points $P, Q, R, S$ in Figure 1 where $R P=k, Q R=(2 i+1) h$, $R S=(2 i-1) h$. If equations $(3)$ and $(4)$ are used to eliminate the time deriva- 


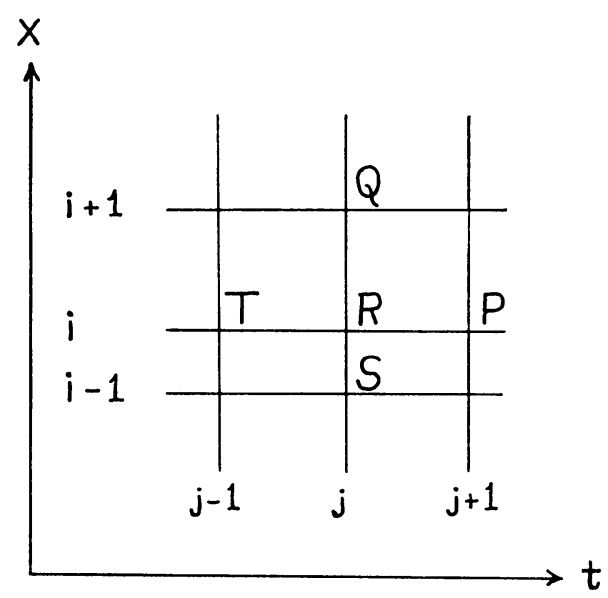

FIG. 1.

tives of $u$, the Taylor expansions of $u$ at points $P, Q, S$ are given by

$$
\begin{aligned}
& u_{i, j+1}=u+q A+q\left(i^{2}+q\right) B+q^{2}\left(2 i^{2}+q\right) C \ldots \\
& u_{i+1, j}=u+(2 i+1) A+\frac{1}{2}(2 i+1)^{2} B+\frac{1}{6}(2 i+1)^{3} C \ldots \\
& u_{i-1, j}=u-(2 i-1) A+\frac{1}{2}(2 i-1)^{2} B-\frac{1}{6}(2 i-1)^{3} C \ldots
\end{aligned}
$$

where $u, A, B, C \cdots$ are the values of $u, h \frac{\partial u}{\partial x}, h^{2} \frac{\partial^{2} u}{\partial x^{2}}, h^{3} \frac{\partial^{3} u}{\partial x^{3}}, \cdots$ at $R$. The linear combination of the values of $u$ at points $P, Q, R, S$ which eliminates $A$ and $B$ is

$$
\begin{aligned}
u_{i, j+1}=[1- & \left.\frac{2 q\left(i^{2}+q-1\right)}{4 i^{2}-1}\right] u_{i, j}+\frac{q\left(2 i^{2}+2 i+2 q-1\right)}{4 i(2 i-1)} u_{i+1, j} \\
& +\frac{q\left(2 i^{2}-2 i+2 q-1\right)}{4 i(2 i-1)} u_{i-1, j}, \quad(i=1,2, \cdots)
\end{aligned}
$$

and the principal part of the truncation error is

$$
-q\left[\left(2 i^{2}+q\right)\left(q-\frac{2}{3}\right)+\frac{1}{6}\right] C,
$$

where $q=k / h$. The coefficients on the right hand side of (5) sum to unity, and except for the coefficient of $u_{i-1, j}$ when $i=1$, are positive for all $i$ if $q \leqq\left(\frac{3}{2}\right)^{1 / 2}$. Thus errors due to round-off do not grow for values of $q$ below this limiting value. It should be noted that if $q=\frac{2}{3}$, the truncation error reduces to $-\frac{c}{9}$ at all points in the field. A modified formula is used to deal with points on the axis. It is

$$
u_{0, j+1}=\frac{1}{4}\left(4-5 q+2 q^{2}\right) u_{0, j}-\frac{2 q}{3}(q-2) u_{1, j}+\frac{q}{12}(2 q-1) u_{2, j},
$$

with a truncation error of $-\frac{q}{3}\left(3_{q}{ }^{2}-5 q+2\right) C$. This is derived by expanding $u_{0, j+1}, u_{1, j}, u_{2, j}$ in terms of $u$ and its derivatives at the point $(0, j)$, and eliminating the time derivatives of $u$ by using (3) and (4). 
If greater accuracy is required, the introduction of a fifth point $T$ where $T R=k$ presents little additional difficulty. Proceeding as before, the five point explicit formula is

$$
\begin{aligned}
u_{i, j+1}= & \frac{q^{2}}{i} \frac{12 i^{4}+12 i^{3}-2 i^{2}-4 i+1-6 q^{2}}{(2 i+1) X} u_{i+1, j} \\
& +\frac{q^{2}}{i} \frac{12 i^{4}-12 i^{3}-2 i^{2}+4 i+1-6 q^{2}}{(2 i-1) X} u_{i-1, j} \\
& +\frac{16\left(4-3 q^{2}\right) i^{4}-8\left(2 q^{2}+3\right) i^{2}+2+24 q^{4}}{\left(4 i^{2}-1\right) X} u_{i, j} \\
& +\frac{4(3 q-2) i^{2}+\left(1-4 q+6 q^{2}\right)}{X} u_{i, j-1}, \quad(i=1,2, \cdots)
\end{aligned}
$$

where

$$
X=4(2+3 q) i^{2}-\left(1+4 q+6 q^{2}\right) .
$$

This time the coefficients sum to unity and are positive for all values of $i$ if $\frac{2}{3} \leqq$ $q \leqq\left(\frac{1}{2}\right)^{1 / 2}$. This stability range is rather more restrictive than necessary, and in fact, as is shown later, there is no significant growth of error in a calculation with $q=$ 0.4 . The principal part of the truncation error in (7) is

$$
\frac{q^{2}}{X}\left[\left(\frac{53}{3}-66 q^{2}\right) i^{4}-\left(\frac{10}{3}-4 q^{2}\right) i^{2}-\left(\frac{1}{6}-q^{2}+12 q^{4}\right)\right] D .
$$

The modified formula used to deal with points on the axis is

$$
\begin{aligned}
u_{0, j+1}=-\frac{2-5 q+3 q^{2}}{2+5 q+3 q^{2}} u_{0, j-1} & +\frac{8-9 q^{2}+6 q^{4}}{2\left(2+5 q+3 q^{2}\right)} u_{0, j} \\
& +\frac{4 q^{2}\left(8-3 q^{2}\right)}{3\left(2+5 q+3 q^{2}\right)} u_{1, j}-\frac{q^{2}\left(1-6 q^{2}\right)}{6\left(2+5 q+3 q^{2}\right)} u_{2, j}
\end{aligned}
$$

with a truncation error of

$$
\frac{q^{2}}{18\left(2+5 q+3 q^{2}\right)}\left(24-117 q^{2}+108 q^{4}\right) D .
$$

This is derived in a similar manner to $(6)$, with the incorporation of the additional node $(0, j-1)$.

4. Implicit Formulas. It is a general feature of finite difference methods that the maximum time interval permissible in a numerical solution of the heat flow equation can be increased by the use of implicit rather than explicit formulas.

Returning to Figure 1, the optimum four point implicit formula involving the values of $u$ at the points $Q, R, S, T$ is

$$
\begin{aligned}
{\left[1+\frac{2 q\left(i^{2}-q-1\right)}{4 i^{2}-1}\right] u_{i, j}+} & \frac{q\left(-2 i^{2}-2 i+2 q+1\right)}{4 i(2 i+1)} u_{i+1, j} \\
& +\frac{q\left(-2 i^{2}+2 i+2 q+1\right)}{4 i(2 i-1)} u_{i-1, j}=u_{i, j-1} .
\end{aligned}
$$


This is obtained in a similar manner to (5) and the principal part of the truncation error is

$$
q\left[\left(2 i^{2}-q\right)\left(q+\frac{2}{3}\right)-\frac{1}{6}\right] C .
$$

The values of $u$ at points on the axis are obtained from (6) with $j-1$ replacing $j$. The implicit formula (9) is stable if the latent root of minimum modulus of the matrix

$$
\left[\begin{array}{lll}
1-\frac{2 q^{2}}{3} & \frac{q(2 q-3)}{12} & \\
\frac{q(2 q-3)}{24} 1+\frac{2 q(3-q)}{15} & \frac{q(2 q-11)}{40} & \\
\frac{q\left(2 q-2 i^{2}+2 i+1\right)}{4 i(2 i-1)} & 1+\frac{2 q\left(i^{2}-1-q\right)}{4 i^{2}-1} & \frac{q\left(2 q-2 i^{2}-2 i+1\right)}{4 i(2 i+1} \\
& \frac{q\left(-2 N^{2}+2 N+1+2 q\right)}{4 N(2 N-1} & 1+\frac{2 q\left(N^{2}-i-q\right)}{4 N^{2}-1}
\end{array}\right]
$$

exceeds unity, where $N^{2}=1 / 4 h$. This condition imposes a restriction on $q$, which depends on $N$ (or $h$ ). The algorithm of unknown origin (Richtmyer [4], p. 102) used frequently to solve the set of equations at each time step in an implicit procedure of this type also imposes a restriction on $q$. In fact it is $q \leqq\left(\frac{3}{2}\right)^{1 / 2}$, a condition which is independent of $N$, and corresponds exactly with the stability condition on $q$ for the explicit scheme (5). Thus it appears that the implicit scheme (9) may have no advantage over (5) either in length of permissible time step or in accuracy, since the truncation errors are similar for the two formulas. If, however, the set of equations at each time step is solved satisfactorily by a method such as simple elimination which does not impose a restriction on the size of $q$ the implicit scheme (9) may well have an advantage in length of permissible time step over the explicit scheme (5).

The implicit scheme used by Albasiny is the six point Crank-Nicolson replacement of (1). It is

$$
\begin{aligned}
\frac{p}{2}\left(1+\frac{1}{2 i}\right) u_{i+1, j+1} & -(1+p) u_{i, j+1}+\frac{p}{2}\left(1-\frac{1}{2 i}\right) u_{i-1, j+1} \\
= & -\frac{p}{2}\left(1+\frac{1}{2 i}\right) u_{i+1, j}-(1-p) u_{i, j}-\frac{p}{2}\left(1-\frac{1}{2 i}\right) u_{i-1, j}
\end{aligned}
$$

where $p=k / h_{0}{ }^{2}$, and the spacings in the $r$-and $t$-directions are given by $r=i h_{0}(i=1,2,3, \cdots)$ and $t=j k(j=1,2,3, \cdots)$ respectively. This is not an optimum formula and cannot be obtained by the methods advocated in the present paper. It is impossible to quote the principal part of the truncation error of (10) in a meaningful form, because as shown by (2), all time derivatives of $u$ involve at least $\frac{\partial u}{\partial r}$ and $\frac{\partial^{2} u}{\partial r^{2}}$. For what it is worth, we quote

$$
\frac{p}{6 i} h_{0}^{3} \frac{\partial^{3} u}{\partial r^{3}}+\frac{p}{8 i} h_{0} k^{2} \frac{\partial^{3} u}{\partial r \partial t^{2}}-\frac{1}{24} k^{3} \frac{\partial^{3} u}{\partial t^{3}}
$$


TABLE I

Errors on axis $\left(\times 10^{9}\right)$

\begin{tabular}{|c|c|c|c|c|c|c|c|c|c|}
\hline \multirow{3}{*}{$t$} & \multicolumn{4}{|c|}{ Crank-Nicolson } & \multicolumn{3}{|c|}{4 Point } & \multicolumn{2}{|c|}{5 Point } \\
\hline & \multicolumn{4}{|c|}{$p$} & \multicolumn{3}{|c|}{$q$} & \multicolumn{2}{|c|}{$q$} \\
\hline & 0.4 & 0.8 & 1.2 & 4.0 & 0.4 & $\frac{2}{3}$ & 0.8 & 0.4 & 0.8 \\
\hline $\begin{array}{l}0.016 \\
0.032 \\
0.048 \\
0.064 \\
0.080 \\
0.096 \\
0.192 \\
0.384 \\
0.768 \\
0.960 \\
1.920 \\
3.840 \\
7.680\end{array}$ & $\begin{array}{r}+2,241 \\
+4,731 \\
+7,367 \\
+10,087 \\
+12,852 \\
+15,636 \\
+32,130 \\
+61,546 \\
+111,763 \\
+131,325\end{array}$ & $\begin{array}{r}+4,678 \\
+10,036 \\
+15,589 \\
+32,073\end{array}$ & $\begin{array}{r}+15,508 \\
+31,977 \\
+62,179 \\
+130,787 \\
+188,798 \\
+191,655 \\
+96,771\end{array}$ & $\begin{array}{r}+124,648 \\
+180,108 \\
+182,946 \\
+92,391\end{array}$ & $\begin{array}{r}+418 \\
+663 \\
+798 \\
+864 \\
+888 \\
+884 \\
+626 \\
-305 \\
-3,282 \\
-5,127 \\
-13,818 \\
-19,330 \\
-11,209\end{array}$ & $\begin{array}{r}+1,313 \\
+1,905 \\
+1,928 \\
+967\end{array}$ & $\begin{array}{l}-2,489 \\
-2,942 \\
-3,220 \\
-3,593\end{array}$ & $\begin{array}{r}0 \\
0 \\
0 \\
0 \\
0 \\
0 \\
+4 \\
+8 \\
+18 \\
+23 \\
+43 \\
+52 \\
+29\end{array}$ & $\begin{array}{r}+20 \\
+11 \\
-12 \\
-25 \\
-90 \\
-132 \\
-77\end{array}$ \\
\hline
\end{tabular}

as the truncation error of (10), where no attempt has been made to eliminate time derivatives of $u$ using (2). As might be expected, (10) can be used with larger time steps but is much less accurate than (5) or (7), particularly near the axis.

The authors in fact made several unsuccessful attempts to obtain an implicit replacement of (3) (or (1)) which was not too unwieldy and gave accuracy, comparable with that given by the optimum explicit formulas developed in the present paper, for large time steps. As pointed out previously in discussing formula (9), the maximum permissible time step with implicit formulas may be limited not only by the usual stability considerations but also by the conditions imposed by the method used to solve the set of equations at each time step.

5. Numerical Results. The problem stated previously is solved numerically using optimum explicit formulas (5) and (7) in turn. The results are compared with the theoretical solution and the errors on the axis shown at various values of the time in Table 1. The calculations are carried out for $h=\frac{1}{40 \overline{0}}$, and so eleven points including the nodes on the axis and the perimeter are sufficient to cover the region in the $x$-direction. The problem is also solved using the Crank-Nicolson six point replacement of (1) with eleven points including the axis and perimeter nodes covering the region in the $r$-direction $\left(h_{0}=\frac{1}{10}\right)$. The errors on the axis are also shown in Table 1.

The time range for each calculation is sufficiently large for the error on the axis to have reached a maximum value, and the calculations are carried out for several values of the mesh ratio $q$ (or $p$ ). The maximum errors are approximately

$\begin{array}{lrl}\text { Crank-Nicolson } & 2 \times 10^{-4} & (p=0.4,0.8,1.2,4.0) \\ \text { 4 Point Explicit } & 2 \times 10^{-5} & (q=0.4) \\ & 2 \times 10^{-6} & \left(q=\frac{2}{3}\right) \\ & 8 \times 10^{-6} & (q=0.8) \\ 5 \text { Point Explicit } & 5 \times 10^{-8} & (q=0.4) \\ & 1.5 \times 10^{-7} & (q=0.8)\end{array}$


There is little doubt that in a problem of this type where there is no singularity in the boundary data, the optimum explicit formulas derived in the present paper are more accurate and easier to apply than the Crank-Nicolson formula. Also, in solving heat conduction problems by finite difference methods, the maximum errors occur at small values of the time, and so there is no disadvantage in using explicit formulas with small time steps. The advantage of the Crank-Nicolson formula in permitting larger time steps cannot be utilized when accuracy is required in the time range near the start of the computation, where the errors are comparatively large.

The five point formula ( 7 ), is a three-level formula and so starting values are required on $t=k$, as well as on $t=0$. These were obtained from the theoretical solution, although in most problems, the theoretical solution is not available. In such cases, the values on $t=k$ are obtained from the four point explicit scheme (5) together with a suitably reduced value of $h$.

Finally, it should be pointed out that it has not yet been found possible to use formulas (5) and (7) to give results of an accuracy comparable with that achieved in the present paper when used to solve a problem with a discontinuity in the boundary data. Such a problem is Albasiny's problem of a cooling spinning thread line where $F(0) \neq 0$. The discontinuity in $\frac{\partial u}{\partial r}$ at $r=1, t=0$ ensures that all finite difference solutions lose accuracy in the neighborhood of the discontinuity; this loss of accuracy is transmitted across the field with time before eventually dying out.

6. Acknowledgment. The calculations were carried out on the Stantec Zebra computer of Queen's College, Dundee.

Mathematics Department

St. Salvator's College

St. Andrews, Scotland

Mathematics Department

University of California

Berkeley

1. E. L. Albasiny, "On the numerical solution of a cylindrical heat-conduction problem," Quart. J. Mech. Appl. Math., v. 13, 1960, p. 374-384.

2. R. P. Pearce \& A. R. Mitchell, "On finite difference methods of solution of the transport equation," Math. Comp. v. 16, 1962, p. 155-169.

3. A. R. Mitchell \& R. P. Pearce, "High accuracy difference formulae for the numerical solution of the heat conduction equation," The Computer Journal, v. 5, 1962, p. 142-147.

4. R. D. Richtmyer, Difference Methods for Initial-Value Problems, Interscience Publishers Inc., New York, 1957. 